\title{
Coxiella burnetii, the agent of $Q$ fever in Brazil: its hidden role in seronegative arthritis and the importance of molecular diagnosis based on the repetitive element IS1111 associated with the transposase gene
}

\author{
Tatiana Rozental' ${ }^{1+}$, Luis Filipe Mascarenhas ${ }^{2}$, Ronaldo Rozenbaum ${ }^{2,3}$, Raphael Gomes ${ }^{1}$, \\ Grasiely Souza Mattos', Cecília Carlos Magno ${ }^{4}$, Daniele Nunes Almeida', \\ Maria Inês Doria Rossi ${ }^{1}$, Alexsandra RM Favacho', Elba Regina Sampaio de Lemos ${ }^{1}$ \\ ${ }^{1}$ Laboratório de Hantaviroses e Rickettsioses, Instituto Oswaldo Cruz-Fiocruz, Rio de Janeiro, RJ, Brasil ${ }^{2}$ Hospital Servidores do Estado, \\ Rio de Janeiro, RJ, Brasil ${ }^{3}$ Hospital Samaritano, Rio de Janeiro, RJ, Brasil ${ }^{4}$ Hospital Copa-D’Or, Rio de Janeiro, RJ, Brasil
}

Coxiella burnetii is the agent of $Q$ fever, an emergent worldwide zoonosis of wide clinical spectrum. Although C. burnetii infection is typically associated with acute infection, atypical pneumonia and flu-like symptoms, endocarditis, osteoarticular manifestations and severe disease are possible, especially when the patient has a suppressed immune system; however, these severe complications are typically neglected. This study reports the sequencing of the repetitive element IS1111 of the transposase gene of $\mathrm{C}$. burnetii from blood and bronchoalveolar lavage (BAL) samples from a patient with severe pneumonia following methotrexate therapy, resulting in the molecular diagnosis of $Q$ fever in a patient who had been diagnosed with active seronegative polyarthritis two years earlier. To the best of our knowledge, this represents the first documented case of the isolation of C. burnetii DNA from a BAL sample.

Key words: Q fever - bronchoalveolar lavage - molecular analysis - seronegative polyarthritis - methotrexate

Q fever is caused by Coxiella burnetii, a small obligate intracellular Gram-negative bacterium of the order Legionellales (Stein et al. 1993). C. burnetii has a variety of hosts, including many vertebrates and ticks. The transmission of $C$. burnetii in humans typically occurs via the inhalation of contaminated aerosols from fresh or desiccated urine, faeces, milk and birth products; less commonly, transmission occurs through the consumption of raw milk and milk products (Maurin \& Raoult 1999, Tissot-Dupont \& Raoult 2008).

The incubation period for $C$. burnetii is variable, depending on the infecting dose and the health status of the patient. Primary infection with $C$. burnetii is commonly asymptomatic. In the symptomatic acute disease, flulike syndrome, pneumonia and hepatitis are considered to be the classic presentations, but rash, pericarditis, myocarditis, aseptic meningitis, encephalitis and osteomyelitis have also been described. Fatalities are rare and are typically associated with other debilitating health conditions. In most patients, the acute disease either has a self-limited course or responds to appropriate therapy (Maurin \& Raoult 1999, Tissot-Dupont \& Raoult 2008). Following the acute phase of $\mathrm{Q}$ fever, a small number of

Financial support: FIOCRUZ/MS

Partial data were presented at the 6th International Meeting on Rickettsiae and Rickettsial Diseases in June 2011 as a poster. It was published as an abstract in the Abstract Book (2011) (P093), in Clin Microbiol Infect p. 72.

+ Corresponding author: rozental@ioc.fiocruz.br

Received 28 October 2011

Accepted 12 April 2012 patients present symptoms of $Q$ fever fatigue syndrome (QFS), characterised by headache, joint and muscle pain and fatigue (Ayres et al. 1996, Raoult et al. 2000, Pappas et al. 2003, Arashima et al. 2004, Hickie et al. 2006, Ledina et al. 2007). Chronic Q fever occurs in $1 \%$ of infected patients months or years after the initial infection. The most common form of chronic Q fever is endocarditis, which is typically associated with an underlying valvulopathy and immunosuppression. Less-common presentations of chronic Q fever include granulomatous lesions in the bones, joints, liver, lung, testis and soft tissues (Ralph et al. 2007, Tissot-Dupont \& Raoult 2008). Studies have shown that $C$. burnetii can be reactivated during pregnancy and in patients with immunosuppression. Studies suggest that $C$. burnetii persists after most instances of acute Q fever, regardless of clinical status and that immunogenic variation in the response to persistent infection can lead to cytokine dysregulation (Penttila et al. 1998, Harris et al. 2000, Pappas et al. 2003, Ledina et al. 2007).

Although impaired T-cell immunity in patients with human immunodeficiency virus (HIV), cancer, lymphoma and pregnancy has been associated with the failure to eradicate $C$. burnetii and progression to the chronic disease, there are no previous reports of an association between Q fever and the use of methotrexate (MTX) for immunosuppressive therapy (Maurin \& Raoult 1999, Nausheen \& Cunha 2007). Low doses of MTX have been used effectively for various rheumatic and nonrheumatic diseases and opportunistic infections caused by Histoplasma, Cryptococcus, Nocardia, Mycobacterium, human herpesvirus, the hepatitis virus and the Epstein-Barr virus in patients receiving MTX therapy (Boerbooms et al. 1995, Feng et al. 2004, Angit \& Daly 2009, McLean-Tooke et al. 2009). This article shows that 
Q fever, confirmed by molecular and serological analysis, is a probable complication of immunosuppression resulting from the use of MTX.

On 13 October 2010, a 33-year-old man from Rio de Janeiro, Brazil, was admitted to a private hospital with symptoms of a fever, myalgia and a dry cough. Notably, the patient's medical history showed that he had been diagnosed with seronegative polyarthritis two years earlier and treated with immunosuppressive therapy using MTX 40 days before hospitalisation. Initial tests revealed that the patient had a white blood cell count of $5 \times 10^{9} \mathrm{~L}^{-1}$, with $18 \%$ band forms and C-reactive protein (CRP) levels of $310 \mathrm{mg} \mathrm{L}^{-1}\left(>10 \mathrm{mg} \mathrm{L}^{-1}\right)$. The chest radiograph showed consolidation of the lower lobe of the right lung. MTX therapy was suspended and treatment with amoxicillin/ clavulanic acid and azithromycin was started. The patient did not respond to antibiotics and five days later, his chest $\mathrm{X}$-ray showed an increase in bilateral pulmonary infiltrates. His condition continued to deteriorate, with progressive hypoxia requiring an elective intubation. In the intensive care unit, additional tests revealed a total leukocyte count of $10.48 \times 10^{9} \mathrm{~L}^{-1}$, a CRP level of $349 \mathrm{mg} \mathrm{L}^{-1}$ and a normal transthoracic echocardiogram. The antibiotic regimen was changed to meropenem, amphotericin, ceftriaxone, vancomycin and doxycycline. The blood and bronchoalveolar lavage (BAL) samples were analysed using culture techniques and were negative for the usual bacteria and fungi. Additional laboratory tests - serological and/or molecular diagnostic assays - were also negative for HIV, cytomegalovirus, Human adenovirus, human herpesvirus, Rickettsia spp and Bartonella spp.

Two serum samples, collected on days 7 and 27 of the illness, were tested for $C$. burnetii using a commercial indirect immunofluorescence assay (IFA) for class-specific $\mathrm{IgM}$ and $\mathrm{IgG}$, cut off $=64$ (Panbio, Brisbane, AU). Titres of IgM antibodies against $C$. burnetii (phase II) were detected in the serum samples at a titre of 64 and Q fever was confirmed in the second serum sample, with C. burnetii serum titres of 512 for phase I and II IgG. The C. burnetii DNA sequences were detected by polymerase chain reaction (PCR) performed on the serum and BAL samples collected on day 7 of the illness, using the primers QBT-1 (5'-TATGTATCCACCGTAGCCAGC-3') and QBT-2 (5'-CCCAACAACACCTCCTTATC-3'), which amplify a $687 \mathrm{bp}$ fragment of the repetitive element IS1111 of a heat shock protein gene ( $h t p \mathrm{AB}$ transposase) (Hoover et al. 1992). The PCR was repeated without the positive control and the results were confirmed. The amplicons were purified and the sequencing was performed using an ABI PRISM BigDye Terminator v.3.1 Cycle Sequencing kit (Applied Biosystems, Foster City, CA, USA). The resulting partial sequences were subjected to BLAST analysis and the nucleotide sequence generated from the BAL sample (585 bp) showed 99\% sequence identity with the homologous gene fragment of the $h t$ pAB gene from C. burnetii RSA 331, complete genome (GenBank accession JF968204).

A detailed epidemiological history was obtained and exposure to dogs and cats, some of them parturient, was identified during the two years preceding the illness, when the patient moved to a new office located in front of a veterinary clinic. During this time, after a severe flu-like disease, the patient developed seronegative polyarthritis.

The patient recovered and was discharged from the hospital and followed up as an outpatient. Doxycycline was administered to the patient for 14 days and this treatment was extended for an additional week because there was a recurrence of fever. During the outpatient follow-up, the patient developed no signs of $\mathrm{Q}$ fever and fully recovered from the osteoarticular manifestations. Subsequently, serum samples were obtained six-nine months after the patient's discharge from the hospital and additional IFA analysis was performed. The serum collected on month 6 was reactive to the phase I and II $C$. burnetii antigen, with IgG titres of 2.048 (phase I and II) and phase II IgM titres of 64 . In the serum sample taken on month 9, IFA showed C. burnetii phase I and II IgG titres of 512 and the IgM titre was negative.

Although the patient's symptoms had resolved, the IgG antibody titre against the C. burnetii phase I and II antigen remained elevated for nine months after successful treatment with doxycycline. Because the most important criterion in monitoring a recurrence of $\mathrm{Q}$ fever would be an increase in the IgG titres (4-fold), the patient should be monitored for this possibility every three months.

In our report, the initial etiological diagnosis of a lobar pneumonia in this patient was limited to communityacquired pneumonia, more specifically the pneumonia caused by pneumococcus. Spotted fever and Q fever, among other infectious diseases, were also initially considered because he had a recent history of animal exposure during an international trip to South Africa.

Initially, the patient received amoxicillin/clavulanic acid and azithromycin, but remained febrile and developed hypoxia and acute bilateral pulmonary infiltrates. Therefore, the antibiotic regimen was changed and treatment with broad-spectrum antibiotics in conjunction with doxycycline resulted in a rapid clinical improvement. Studies have shown that patients treated with azithromycin remained febrile for multiple days compared with one day for patients treated with doxycycline (Ralph et al. 2007, Dijkstra et al. 2010).

Although there are reports of opportunist pulmonary infections in patients with defective cellular immunity who were treated with low doses of MTX, these cases are unusual and possibly underdiagnosed; in these cases, as was relevant in this study, MTX therapy was discontinued. We reported a patient with a history of seronegative polyarthritis whose immunosuppressive MTX treatment likely induced the reactivation of C. burnetii and led to a severe pneumonia.

Considering that the $C$. burnetii infection was likely acquired in his workplace and the doxycycline therapy resulted in a clinical improvement with complete resolution of the polyarthritis, could this seronegative rheumatologic disease have been diagnosed as QFS before the MTX therapy? There are studies that support this hypothesis (Harris et al. 2000, Raoult et al. 2000, Pappas et al. 2003, Arashima et al. 2004, Hickie et al. 2006, Ledina et al. 2007).

To our knowledge, this paper reports the first documented case of $C$. burnetii present in a BAL sample obtained from a patient after MTX therapy. At present, $C$. 
burnetii has only been isolated from urine, semen and bone marrow samples obtained from patients with Q fever (Kruszewska et al. 1996, Maurin \& Raoult 1999, Marmion et al. 2005).

$\mathrm{Q}$ fever is not a common diagnosis and it may easily be overlooked in immunocompetent or immunodeficient individuals and pregnant women, being misinterpreted as several other infectious diseases. Q fever should be added to the list of diseases known as probable complications of immune suppression and PCR based on the repetitive element IS1111 of the transposase gene could be used as an important diagnostic method for Q fever.

\section{REFERENCES}

Angit C, Daly BM 2009. Disseminated varicella zoster infection in a patient with rheumatoid arthritis treated with methotrexate. Clin Exp Dermatol 34: 453-454.

Arashima Y, Kato K, Komiya T, Kumasaka K, Matsukawa Y, Murakami M, Takahashi K, Ikeda T, Arakawa Y 2004. Improvement of chronic nonspecific symptoms by long-term minocycline treatment in Japanese patients with Coxiella burnetii infection considered to have post- $\mathrm{Q}$ fever fatigue syndrome. Intern Med 43: 49-54.

Ayres JG, Smith EG, Flint N 1996. Protracted fatigue and debility after acute Q fever. Lancet 347: 978-979.

Boerbooms AM, Kerstens PJ, van Loenhout JW, Mulder J, van de Putte LB 1995. Infections during low-dose methotrexate treatment in rheumatoid arthritis. Semin Arthritis Rheum 24: 411-421.

Dijkstra F, Riphagen-Dalhuisen J, Wijers N, Hak E, van der Sande MA, Morroy G, Schneeberger PM, Schimmer B, Notermans DW, van der Hoek W 2010. Antibiotic therapy for acute Q fever in The Netherlands in 2007 and 2008 and its relation to hospitalization. Epidemiol Infect 19: 1-10.

Feng W, Cohen JI, Fischer S, Li L, Sneller M, Goldbach-Mansky R, Raab-Traub N, Delecluse HJ, Kenney SC 2004. Reactivation of latent Epstein-Barr virus by methotrexate: a potential contributor to methotrexate-associated lymphomas. JNCI J Natl Cancer Inst 96: 1691-1702.

Harris RJ, Storm PA, Lloyd A, Arens M, Marmion BP 2000. Longterm persistence of Coxiella burnetii in the host after primary $\mathrm{Q}$ fever. Epidemiol Infect 124: 543-549.
Hickie I, Davenport T, Wakefield D, Vollmer-Conna U, Cameron B, Vernon SD, Reeves WC, Lloyd A 2006. Post-infective and chronic fatigue syndromes precipitated by viral and non-viral pathogens: prospective cohort study. $B M J 333: 1-6$.

Hoover TA, Vodkin MH, Williams JC 1992. A Coxiella burnetii repeated DNA element resembling a bacterial insertion sequence. J Bacteriol 174: 5540-5548.

Kruszewska D, Lembowicz K, Tylewska-Wierzbanowska S 1996. Possible sexual transmission of Q fever among humans. Clin Infect Dis 22: 1087-1088.

Ledina D, Bradarić N, Milas I, Ivić I, Brncić N, Kuzmicić N 2007. Chronic fatigue syndrome after Q fever. Med Sci Monit 13: 88-92.

Marmion BP, Storm PA, Ayres JG, Semendric L, Mathews L, Winslow W, Turra M, Harris RJ 2005. Long-term persistence of Coxiella burnetii after acute primary Q fever. QJM 98: 7-20.

Maurin M, Raoult D 1999. Q fever. Clin Microbiol Rev 12: 518-553.

McLean-Tooke A, Aldridge C, Waugh S, Spickett GP, Kay L 2009. Methotrexate, rheumatoid arthritis and infection risk: what is the evidence? Rheumatology (Oxford) 48: 867-871.

Nausheen S, Cunha BA 2007. Q fever community-acquired pneumonia in a patient with Crohn's disease on immunosuppressive therapy. Heart Lung 36: 300-303.

Pappas G, Akritidis N, Christou L, Mastora M, Tsianos E 2003. Unusual case of reactive arthritis: Leptospira and Coxiella burnetii. Clin Rheumatol 22: 343-346.

Penttila IA, Harris RJ, Storm P, Haynes D, Worswick DA, Marmion BP 1998. Cytokine dysregulation in the post-Q-fever fatigue syndrome. QJM 91: 549-560.

Ralph A, Markey P, Schultz R 2007. Q fever cases in the northern territory of Australia of 1991 to 2006. Commun Dis Intell 31: 222-227.

Raoult D, Tissot-Dupont H, Foucault C, Gouvernet J, Fournier PE, Bernit E, Stein A, Nesri M, Harle JR, Weiller PJ 2000. Q fever 1985-1998. Clinical and epidemiologic features of 1,383 infections. Medicine (Baltimore) 79: 109-123.

Stein A, Saunders NA, Taylor AG, Raoult D 1993. Phylogenic homogeneity of Coxiella burnetii strains as determinated by $16 \mathrm{~S}$ ribosomal RNA sequencing. FEMS Microbiol Lett 113: 339-344.

Tissot-Dupont H, Raoult D 2008. Q fever. Infect Dis Clin N Am 22: 505-514. 